\title{
EXPERIMENTAL FAULT DETECTION AND ACCOMMODATION FOR AN AGRICULTURAL MOBILE ROBOT
}

\author{
K. Østergaard, D. Vinther, M. Bisgaard, \\ R. Izadi-Zamanabadi and J. D. Bendtsen \\ Dept. of Control Engineering, Aalborg University, \\ DK-9220 Aalborg, Denmark, E-mail: \\ $\{k z o e 00, d r v i 00, m b i s 00$, riz,dimon\}@control.auc.dk
}

\begin{abstract}
This paper presents a systematic procedure to achieve fault tolerant capability for a four-wheel driven, four-wheel steered mobile robot moving in outdoor terrain. The procedure is exemplified through the paper by applying on a compass module. Detailed methods for fault detection and fault accommodation for the compass faults are discussed and the results are verified through field tests. Copyright ${ }^{\circledR} 2005$ IFAC
\end{abstract}

Keywords: Fault detection and Accommodation, Fault-tolerant control, Robotics

\section{INTRODUCTION}

This paper reports part of the work carried out in connection with a recently finished project ${ }^{1}$ concerning the construction of a robot for autonomous surveying of agricultural fields. The robot has to navigate to certain waypoints (measurement locations), where digital images of the crops, weeds, etc. can be taken. Information from image analysis will be used for each location to yield a digitized weed map of the field, opening up opportunities for the farmer to adjust the application of fertilizer and pesticides according to the state of the field (precision farming). The robot is equipped with GPS and various onboard sensors, which will not only help in the exact determination of the location where each image is taken, but also provide measurements for an estimation of the robot's position and orientation for tracking control algorithms. The robot is equipped with independent steering and drive

1 API II, a collaboration between various Danish agricultural industry-related companies, the Danish Agricultural Research Center and Aalborg University, Denmark

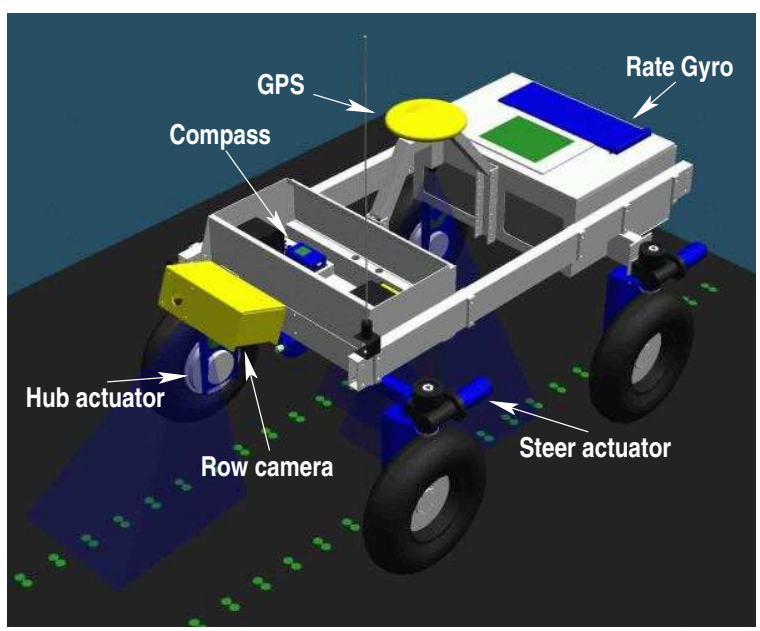

Fig. 1. The API II robot. The dimensions of the robot are $150 \mathrm{~cm}$ (length) by $100 \mathrm{~cm}$ (width) by $107 \mathrm{~cm}$ (height) It weights $226.5 \mathrm{~kg}$.

motors (8 DC motors in total), whose individual controllers are connected to a main computer via a fieldbus. It is thus possible at any given time to set rotation speed or torque references for each motor. The robot is furthermore equipped with various 


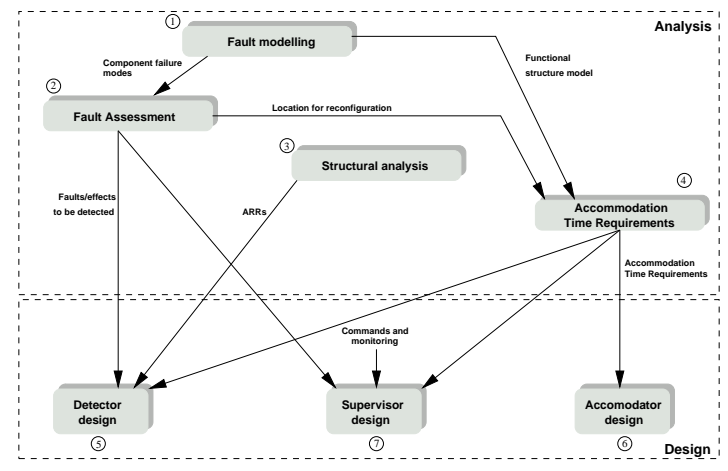

Fig. 2. The (modified) analysis and design procedure to achieve FTC property.

sensors, some of which are shown on Figure 1. A row camera is used for navigating relative to crop rows, a doppler radar is used to measure ground speed, and a compass is mounted to obtain the orientation of the robot. The robot is also equipped with a gyro (not indicated on the figure). An antenna is mounted for the WLAN interface connecting the robot to a centrally positioned computer, which handles generation of the overall plan for the system, storing image data etc.

The model and verification of the robot was presented in a previous paper (Bisgaard et al., 2005). This paper deals with fault tolerant aspects of the robot. Section 2 deals with systematic analysis of the robot in order to identify and classify faults together with establishing detection time requirements. In section 3 a selected compass fault is detected using a statistical method and accommodated for by means of sensor fusion. In section 4 the field test results are used to verify the proposed accommodation strategy. Finally, the concluding remarks are provided in section 5 .

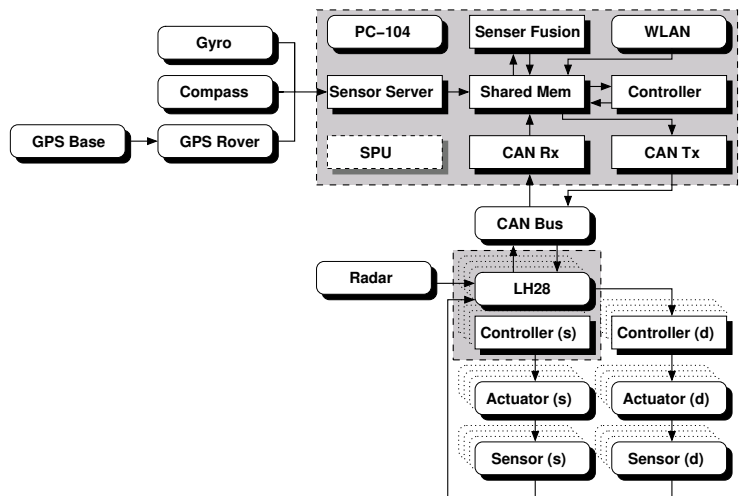

Fig. 3. The API II robot divided into subsystems and modules. Hardware modules with rounded corners and software without. The software that runs on the two different types of computers are indicated with grey boxes.

\section{SYSTEM ANALYSIS}

The main idea of achieving fault-tolerant control (FTC) capability is to handle most important faults, i.e. those that have the most severe impact on the robot's overall objectives. There exist a vast literature concerning hazard analysis methods (toward achieving safety and reliability objectives) within aerospace and automotive industries (see for instance, (DoD, 1980), (DoD, 1981), (IEC, Dec. 1998) which have been inspirentional to our work. The task begins with an analysis of the system w.r.t. achieving fault tolerance by following a stepwise procedure that is shown in figure 2. This is a simplified version of the analysis procedure in (Izadi-Zamanabadi, 1999). In order to perform the analysis, the system is decomposed into clearly identified modules with their physical/functional interfaces as it is illustrated in figure 3 for the API robot.

The steps in the procedure are described and the compass component is been used to illustrate the result of activities in each step.

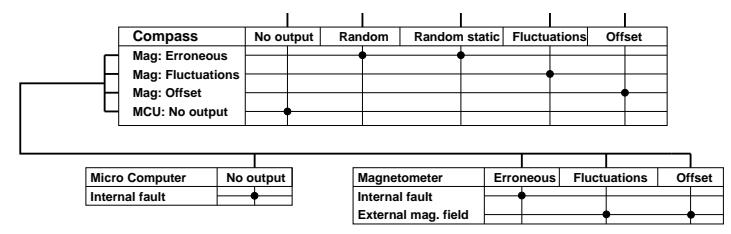

Fig. 4. FMEA scheme for the Compass.

\subsection{Fault modelling}

The system is divided into components (see figure 3 ), which are individually analyzed for possible faults using a proper Hazard analysis technique. For mechanical system the Failure Mode and Effect Analysis (FMEA) technique is been used (International, 1998). The result of FMEA on the compass is shown in figure 4 . The compass is made with solid state magnetic-modules, making it rugged and reliable. A likely fault is disturbance originating from externally generated magnetic field (such as high voltage lines or motors on the robot) and can result in offset or large fluctuations.

\subsection{Fault assessment}

This step includes fault propagation analysis through the system levels, severity assessment for each fault, and causal relation analysis. The result would be a set of identified severe faults that require detection and handling.

Fault assessment is performed by using a Severity Occurrence (SO) index. Each fault is assessed 
with respect to severity and occurrence with a number from 1 to 10 where 10 is the highest rated i.e. severity 10 is very severe and occurrence 10 is very likely to happen. The SO index is then obtained by multiplying the severity index with the occurrence index (International, 1998).

Each fault has been evaluated by means of simula-

Table 1. Severity and occurrence analysis of the compass.

\begin{tabular}{lccc}
\hline Effect & Severity & Occurrence & SO \\
\hline No output & 9 & 2 & 18 \\
Random output & 9 & 2 & 18 \\
Random static output & 9 & 2 & 18 \\
Fluctuations - small & 4 & 8 & 32 \\
Fluctuations - large & 8 & 4 & 32 \\
Offset - small & 2 & 8 & 16 \\
Offset - large & 9 & 4 & 36 \\
\hline
\end{tabular}

tion and actual implementation on the robot. The fault assessment result including the SO index for the compass is shown in table 1.

2.2.1. Fault simulation and injection The faults in the table are evaluated by means of either simulation or fault injection on the actual robot. Simulation reveals that robot can reach the waypoints, but with an error on the orientation and the position. Already at an offset of $\pm 10^{\circ}$ the
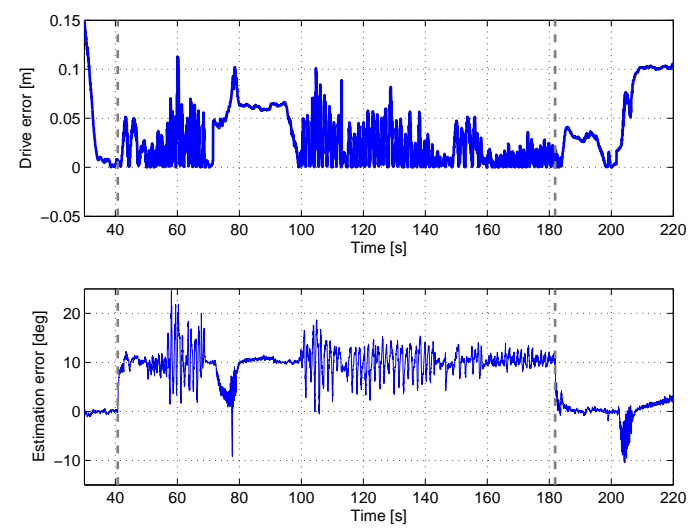

Fig. 5. An offset of $+10^{\circ}$ on the compass results in a small deviation on the position and an error in orientation.

robot will be unable to meet the control objective of keeping the deviation from the path below $5 \mathrm{~cm}$. Simulation shows that throughout the trajectory the robot has an almost constant offset to one side.

Fault injection on the robot yield the same result; the robot tend to move in a non-smooth manner. Figure 5 shows the deviation from the course (in meter) and the orientation (in deg.)

The simulations and experiments illustrate the impact of faults on the control objectives which are: - the robot must be able to estimate position and orientation with a precision of $\pm 5 \mathrm{~cm}$ and $\pm 5^{\circ}$ and keep the total deviation within a precision of $\pm 10 \mathrm{~cm}$ and $\pm 10^{\circ}$. The results are illustrated in table 2: All the failures are then
Table 2. Results from simulation with injected faults into the compass model.

\begin{tabular}{ll}
\hline Effect & Objectives \\
\hline No output & Not fulfilled \\
Random output & Not fulfilled \\
Random static output & Not fulfilled \\
Fluctuations - small & Fulfilled \\
Fluctuations - large & Not fulfilled \\
Offset - small $\left(<3^{\circ}\right)$ & Fulfilled \\
Offset - large $\left(>3^{\circ}\right)$ & Not fulfilled \\
\hline
\end{tabular}

evaluated to identify the failures which should be acted upon. This also includes identifying the components which could have caused the failure to occur. Failures are classified based on their sever-

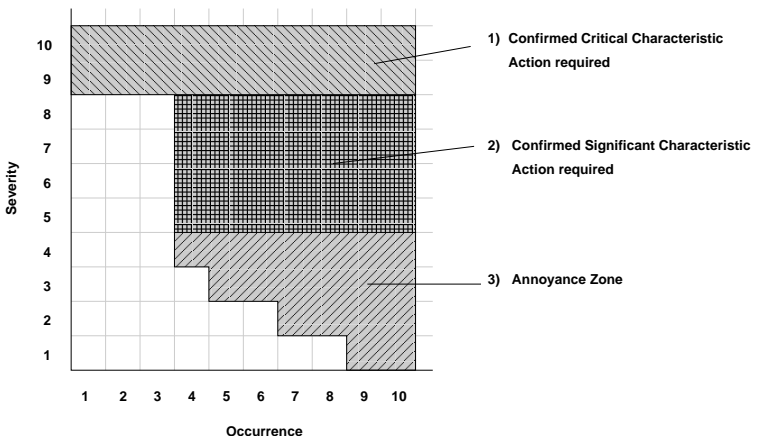

Fig. 6. Action are required with priority 1,2 and 3. Priority 1 and 2 requires action but with priority 3 action is not required but only preferred.

ity and the probable number of occurrence (during the plant's operational life). Figure 6 shows the classification of faults based on their placement on the graph (International, 1998). The graph is divided into three areas which corresponds to the priority of the failures. The choice of priority is based on a trade off between severity and occurrence. The compass failures that fall into priority 1 or 2 are listed in table 3.

Table 3. The failures which should be detected where $\mathrm{S}$ is severity and $\mathrm{O}$ is occurrence.

\begin{tabular}{lcccc}
\hline Effect & S & O & SO & Priority \\
\hline No output & 9 & 2 & 18 & 1 \\
Random output & 9 & 2 & 18 & 1 \\
Random static output & 9 & 2 & 18 & 1 \\
Small fluctuations & 4 & 8 & 32 & 3 \\
Large fluctuations & 8 & 5 & 40 & 2 \\
Small offset & 2 & 8 & 16 & 3 \\
Large offset & 9 & 4 & 36 & 1 \\
\hline
\end{tabular}

\subsection{Structural analysis}

A general framework for an analysis of diagnostic feasibility and possibility is the structural approach (Declerck and Staroswiecki, 1991), (IzadiZamanabadi and Staroswiecki, 2000). The main objective of the structural approach is to identify the parts/ subsystems in the plant that contain redundant information, which in turn can be 
faults by using appropriate methods. The structure model of a system does not depend on detailed knowledge of parameters or dynamic relations within the plant, only relation between the constraints (i.e. diff. equations, algebraic eqs., rules ) and the variables are considered. It shall be noticed that the analysis is performed on the complete nominal system (with no fault).

\subsection{Accommodation time requirement}

Each fault is assigned an accommodation time, in order to continue operation within the objectives, and a critical time requirement which will define when the fault will develop to a system failure.

The fault effect 'no output' from the compass (due to an internal fault in the compass micro controller) causes an offset in the estimated orientation. After around 10 seconds the offset could be more than $10^{\circ}$ (during extreme manoeuvres). The 40 seconds are set as the critical limit for extreme manoeuvering situation as the worst case.

Random output (cause: internal fault in the magnetometer) can cause the robot to move away from the trajectory and it could result in overload in the electrical part of the motion control system. The accommodation time is set at $5 \mathrm{sec}$. and the critical time is set at $10 \mathrm{sec}$.

The fault effect "random static output" (cause: an internal fault in the magnetometer) causes the sensor fusion to function without the compass whereby it can be compared to 'no output' of the compass. The accommodation time is therefore set at 10 sec. and the critical time is set at 40 sec..

Small fluctuations in the compass (cause: an external magnetic field) could result in minor changes in the performance of the robot, but the robot will still be able to fulfil the objectives. The accommodation time is therefore set at $10 \mathrm{sec}$. and critical time is not set. Large fluctuations in the
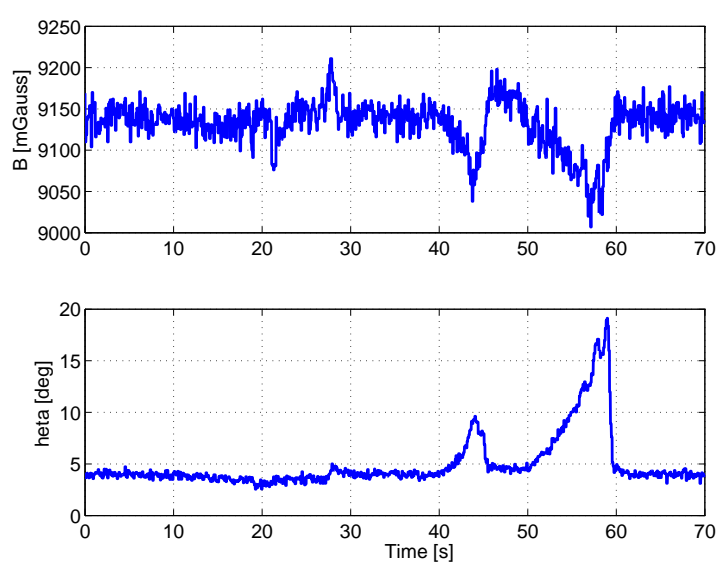

Fig. 7. Top: Total magnetic field when injecting fault. Bottom: Heading measurement. compass (cause: large external magnetic field) can result in the robot being unable to fulfil the objectives. However, the robot will be able to follow the trajectory with the fault but, depending on how large the fluctuations are, the robot will obtain an offset in orientation. The accommodation time is therefore set at 5 sec. as it is assumed that the external magnetic field will increase gradually and the critical time is set at $20 \mathrm{sec}$. .

A large offset on the compass can be caused by an powerful external magnetic field and could result in significant deviations from the trajectory. The accommodation time is therefore set at 5 seconds again because the external magnetic field is expected to increase gradually and the critical time is set to 20 seconds.

\section{FAULT DETECTION AND ACCOMMODATION IN PRACTICE}

\subsection{Fault detection}

An external magnetic field (cause: nearby highvoltage power lines) has different effects on the compass depending on whether it is parallel to or perpendicular to the robot movement.

The general effect of the fault is that the total magnetic field (the sum of the three axes) is changed. The method for fault detection is therefore to detect this change in the total magnetic field strength. This total magnetic field depends on the amount of ferromagnetic material on the robot and the current in the power lines or the actuators. The compass is calibrated so that these currents and magnetic materials do not affect the measurement of the heading significantly, but the total magnetic field will be affected slightly.

To perform the fault detection the tolerable levels for the total magnetic field strength needs to be identified. This is tested by moving a magnetic material near the compass and observe at which total magnetic field strength the heading is changed from the correct magnetic heading. The result from this test is shown in figure 7 where it can be seen that a change in the magnetic field of approximately 100 mGauss cause a change in the heading of approximately 10 degrees when injected to the east of the magnetometer, i.e. perpendicular to the magnetic field of the earth. This fault need to be detected relatively fast and it has been chosen to design for a detection time of one second for a fault causing a change in magnetic field of 100 mGauss. Our field tests underscore the importance of instrument calibration in order to obtain reliable fault diagnosis information.

Applying a 100 mGauss magnetic field in the field test causes a fault in orientation of approx. $10^{\circ}$ which needs to be detected relatively fast. 

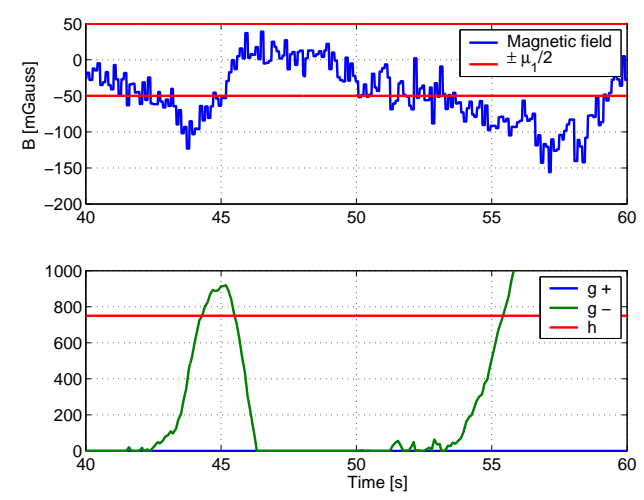

Fig. 8. Top: Magnetic field after recalibration. Horizontal lines mark threshold between zero and alternative hypothesis. Bottom: decision function.

Hence, the alternative hypothesis has been chosen to \pm 100 mGauss and the detection time has been chosen to one second for a 100 mGauss fault. Assuming that the noise is white, a CUSUM algorithm (Basseville and Nikiforov, 1993, page 3841 ) is chosen. The threshold is designed, using Siegmund's approximation (see (Basseville and Nikiforov, 1993, page 187)), for a detection time of 1 second which yields a threshold of 750 and a very large time between false alarms. The detection algorithm (eqs. 1-3) has been designed for relatively large faults (above 100 mGauss) which means that there will be a slower detection time for faults causing a change in magnetic field smaller than 100 mGauss.

$$
\begin{aligned}
g_{k}^{+} & =\sup \left(g_{k-1}^{+}+\frac{100}{18^{2}}\left(B_{T}-50\right), 0\right) \\
g_{k}^{-} & =\sup \left(g_{k-1}^{-}-\frac{100}{18^{2}}\left(B_{T}+50\right), 0\right) \\
t_{a} & =\min \left\{k:\left(g_{k}^{+} \geq 750\right) \cup\left(g_{k}^{-} \geq 750\right)\right\}
\end{aligned}
$$

$t_{a}$ denotes the alarm time and $B_{T}$ denotes the measurement of total magnetic field (minus the local magnetic field which is a number). Faults below 50 mGauss will not be detected and faults causing a change in magnetic field of 75 mGauss (equal to approximately $5^{\circ}$ when injected from the east) will be detected within 87 samples or $1.75 \mathrm{~s}$. The result from a typical run is shown in figure 8, where the fault is detected within a few seconds. In this test the magnetic source is slowly injected twice and then rapidly removed.

\subsection{Fault Accommodation}

Since it is not possible to repair the compass onboard, the accommodation strategy ${ }^{2}$ would be

\footnotetext{
2 Strictly speaking, we should use the term fault reconfiguration here since there is a difference between fault accommodation and system reconfiguration (see (Blanke et al., 2003)). However, since we use a subset of already in use sensors, i.e. no new sensor is introduced, the term fault accommodation is more appropriate.
}

to remove this measurement from the sensor fusion module and use an estimated orientation by employing the GPS, gyro, steering angle measurements and the kinematic model of the robot as shown in figure 9 . The heading output from the

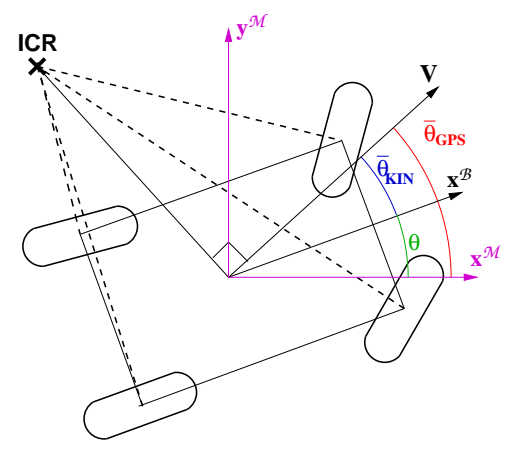

Fig. 9. Estimation of $\theta$ using GPS, gyro and steering angle measurements.

GPS gives an estimate of the velocity heading of the robot which is found as shown in equation 4 .

$$
\bar{\theta}_{G P S}=\tan ^{-1}\left(\frac{\bar{x}_{G P S}(k)-\bar{x}_{G P S}(k-1)}{\bar{y}_{G P S}(k)-\bar{y}_{G P S}(k-1)}\right)
$$

where $\bar{\theta}_{G P S}$ is the velocity heading estimated from GPS measurements, and $k$ is the sampling time.

This heading indicates what can be called the velocity heading of the robot and not the true heading which can be found by using the steering angles. This is done by calculating the velocity vector of the robot in the body frame $(\mathcal{B})$ using the kinematic model to find the Instantaneous Center of Rotation (ICR) and the gyro measurement ((Bisgaard et al., 2004), (Bisgaard et al., 2005)).

$$
\left[\begin{array}{c}
\dot{x}^{\mathcal{B}} \\
\dot{y}^{\mathcal{B}} \\
0
\end{array}\right]=\left[\begin{array}{c}
y_{I C R}^{\mathcal{B}} \\
-x_{I C R}^{\mathcal{B}} \\
0
\end{array}\right] \overline{\dot{\theta}}_{\text {gyro }}
$$

By using this velocity vector it is then possible to calculate the angle between velocity and the robot:

$$
\bar{\theta}_{K I N}=\tan ^{-1}\left(\frac{\dot{y}^{\mathcal{B}}}{\dot{x}^{\mathcal{B}}}\right)
$$

The estimate of $\theta$ in the global reference frame $(\mathcal{M})$ can then be calculated as the difference between the two angles as shown in figure 9 and equation 7 .

$$
\hat{\theta}_{c o m p}^{\mathcal{M}}=\bar{\theta}_{G P S}-\bar{\theta}_{K I N}
$$

An important issue regarding estimation of the heading is the amount of noise on $\bar{\theta}_{G P S}$ which grows larger as the speed of the robot gets smaller. Therefore, it has been chosen to disable the estimate when the speed is less that $0.22 \mathrm{~m} / \mathrm{s}$ and enable it again when the speed is larger than $0.32 \mathrm{~m} / \mathrm{s}$ to provide a hysteresis effect. 


\section{FIELD TEST RESULTS}

The result of a field test run is shown in figure 10 and figure 11. In figure 10 the estimated and real

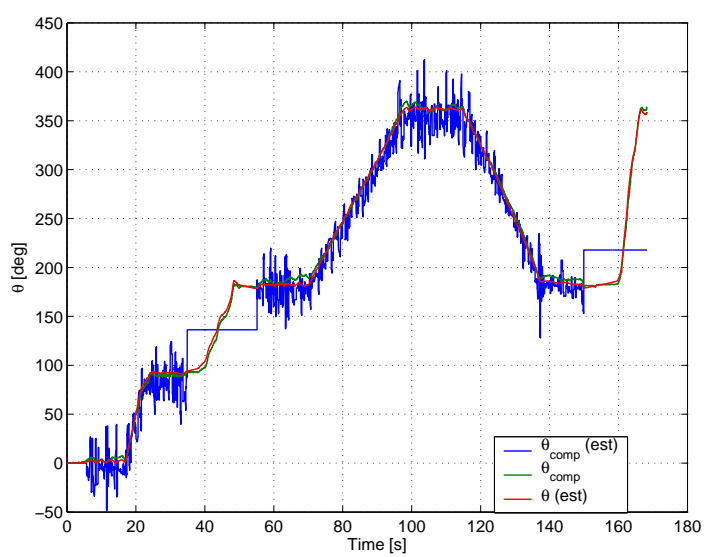

Fig. 10. Verification run: the real and the estimated compass measurement and the estimated $\theta$.

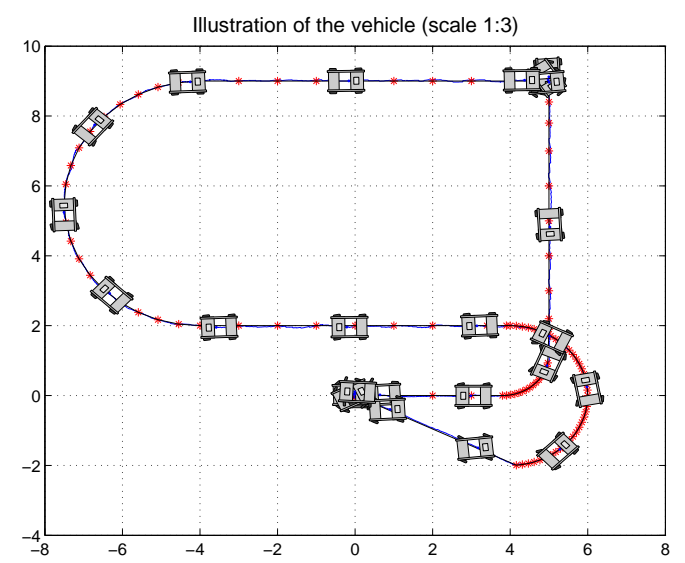

Fig. 11. The robot driving with the estimated compass measurement.

compass measurement is compared with the estimated heading from the sensor fusion. Obviously, the estimated $\theta$ based on the estimated compass measurement nicely follows the actual measurement. Also, the sensor fusion determines the orientation quite well when there are no measurements (due to low speed). Figure 11 shows that the robot drives through the course without any problem using the estimated compass measurement.

\section{DISCUSSION}

Applying the presented procedure for systematic analysis of the system to identify severe faults and their detection possibilities was a necessary step toward achieving our ultimate goal, which is developing a fully autonomous agricultural robot. Fault injection, if possible, provides a realistic picture of fault's effect on the system performance. The prerequisites for achieving good fault diagnosis results are: 1- detailed and verified model, 2-well-calibrated instruments, and 3- robust and simple FDI algorithms.

The CUSUM algorithm showed its usefulness in this regard. However, the Siegmund's approximation provided an overestimated threshold size, that needed modification for actual use.

Our present focus is on the supervision and control using a hybrid control system approach to achieve full autonomy on API II robot.

\section{REFERENCES}

Basseville, M. and I. V. Nikiforov (1993). Detection of Abrupt Changes: Theory and Application. Information and System Science. Prentice Hall. New York.

Bisgaard, M., D. Vinther and K. Z. Ø stergaard (2004). Modelling and fault-tolerant control of an autonomous wheeled robot. Technical report. Control Engineering Department, Aalbog University. Master Thesis.

Bisgaard, M., D. Vinther, K. Z. Østergaard, J. D. Bendtsen and R. I. Zamanabadi (2005). Simulation, sensor fusion and model verification for a mobile robot. In: submitted to International Conference on Robotics and Automation, ICRA 2005. IEEE.

Blanke,

M.,

M. Kinnaert, J. Lunze and M. Staroswiecki (2003). Diagnosis and Fault-tolerant Control. Springer-Verlag.

Declerck, P. and M. Staroswiecki (1991). Characterization of the canonical components of a structural graph for fault detection in large scale industrial plants. In: Proceedings of ECC'91. Grenoble, France. pp. 298-303.

DoD (1980). Procedure for performing a failure mode, effects and criticality analysis. Technical report. Dept. of Defence, NAEC. Lakehurst, NJ, US.

DoD (1981). Reliability modelling and prediction.. Technical report. Dept. of Defence, NAEC. Lakehurst, NJ, US.

IEC (Dec. 1998). IEC 61508 - Functional safety of electrical/lectronic/programmable electronic safety-related systems.

International, Quality Associates (1998). FMEA - Quick Refrence Guide. Quality Associates International. http://www.qualityone.com/services/fmea.cfm.

Izadi-Zamanabadi, R. (1999). Fault-tolerant Supervisory Control - System Analysis and Logic Design. PhD thesis. Dept. of Control Eng., Aalborg University, Denmark.

Izadi-Zamanabadi, R. and M. Staroswiecki (2000). A structural analysis method formulation for fault-tolerant control system design. In: 39th IEEE Conference on Decision and Control. pp. 4901-4902. 